\title{
Long-term influence of associating an antibacterial agent with GIC on bond strength to caries-affected dentin
}

Ana Carolina de Oliveira Beccia, Luana Mafra Martia, Angela Cristina Cilense Zuanon ${ }^{\mathrm{b}}$, Fernanda Lourenção Brighentic, Elisa Maria Aparecida Giro ${ }^{b}$

a PhD student, Faculdade de Odontologia de Araraquara, São Paulo State University (UNESP) Araraquara, SP, Brazil.

${ }^{\mathrm{b}}$ Associate Professor, Faculdade de Odontologia de Araraquara, São Paulo State University (UNESP), Araraquara, SP, Brazil, Department of Orthodontics and Pediatric Dentistry

Assistant Professor, Faculdade de Odontologia de Araraquara, São Paulo State University (UNESP), Araraquara, SP, Brazil, Department of Orthodontics and Pediatric Dentistry.
Corresponding author: Ana Carolina de Oliveira Becci email: acbecci@gmail.com Faculdade de Odontologia de Araraquara - UNESP Rua Humaitá nº 1680 Araraquara, SP, Brasil. CEP: $14801-903$

Tel: +551633016336 fax: +551633016329

Received: April 19, 2017

Accepted: September 14, 2017
Abstract

Aim: To evaluate the bond strength of a GIC associated with chlorhexidine $(\mathrm{CHX})$ to sound and caries-affected dentin, immediately and after six months of storage. Methods: Sixty molars were assigned to two groups of 30 teeth. One had flat dentin surfaces produced and submitted to caries induction to obtain a caries-affected dentin. In the other group dentin was maintained sound. Teeth of each group were randomly reassigned to three subgroups $(n=10)$ according to the concentration of $\mathrm{CHX}$ added to the $\mathrm{GIC}(0 \%, 1 \%$ and $2 \%$ by weight). Two specimens $(1 \mathrm{~mm}$ diameter $\mathrm{x} 1 \mathrm{~mm}$ high) of the same material were constructed on each dentin surface. One was submitted to the microshear bond strength ( $\mu \mathrm{SBS}$ ) test after 24 hours and the other after 6 months of storage in water at $37^{\circ} \mathrm{C}$. Failure modes were analyzed under a stereomicroscope. Bond strength data were analyzed by three-way ANOVA followed by Games-Howell tests for multiple comparisons, and failure modes by the Chi-square test $(a=0.05)$. Results: The $\mu$ SBS values obtained to sound dentin were higher compared with those to caries-affected dentin $(p \leq 0.001)$. In sound dentin, the group with $2 \% \mathrm{CHX}$ showed lower $\mu \mathrm{SBS}$ values compared with $0 \%$ and $1 \% \mathrm{CHX}$ after 24 hours ( $\mathrm{p}=0.005$ and $\mathrm{p}=0.032$ respectively). In caries-affected dentin, after 24 hours, $\mu$ SBS in group with 1\% $\mathrm{CHX}$ was statistically higher than the values in groups with $2 \% \mathrm{CHX}$ after 24 hours ( $p=0.001)$ and $1 \%$ CHX after 6 months ( $p=0.024)$. Irrespective of the condition of substrate, comparisons showed no statistically significant differences between the other groups ( $p \geq 0.053$ ). Cohesive in material and mixed failures prevailed for all groups. Conclusions: The addition of $\mathrm{CHX}$ at concentrations of up to $2 \%$ to the GIC did not affect the bond strength of the material to sound and caries-affected dentin in a long-term evaluation.

Keywords: Glass ionomer cements. Chlorhexidine. Dentin 


\section{Introduction}

Contemporary restorative dentistry concepts characterize a less invasive approach to the treatment of carious lesions ${ }^{1}$. Studies have recommended that infected dentin should be removed and caries-affected dentin should be preserved. These substrates show outstanding differences in their characteristics. Infected dentin is defined as the necrotic surface area of a highly demineralized substrate ${ }^{2-4}$ in which there are degenerated collagen fibrils and presence of a bacterial biomass ${ }^{2,4}$. The affected dentin, capable of remineralization, is considered a variation of reactive dentin produced in response to soft stimuli such as caries, presenting small changes in the cross-linking of its collagen fibrils ${ }^{2-4}$.

In this scenario, the use of glass ionomer cements (GICs) is interesting because of their capacity for releasing ions - especially fluoride ${ }^{5-7}$ - that may improve the remineralization of caries-affected dentin ${ }^{5}$. This feature along with low initial pH of GICs gives them their antibacterial property. The release of ions from GICs, however, decreases rapidly over time making it interesting to associate this material with antimicrobial compounds to reduce recurrent caries at restoration margins; inhibit biofilm formation on the restored surface; reduce the number of microorganisms in oral cavity fluids, and inhibit microbial growth under the restoration ${ }^{8,9}$. Previous in vitro studies have shown that the incorporation of chlorhexidine (CHX) into GICs reduces Streptococcus mutans, Lactobacillus spp, Candida albicans and Actinomyces naes/undii-11. However, its antibacterial effect is concentration-dependent and in high concentrations $\mathrm{CHX}$ may interfere with the mechanical properties of $\mathrm{GIC}^{10}$.

As the longevity of tooth restorations is partly related to mechanical properties, among them a good bond to tooth structures, the antibacterial agent should be added at a concentration that will not impair these properties of GICs ${ }^{11,12}$. To date, there are few studies that have investigated how GIC bonds are affected by time ${ }^{13,14}$ or by the presence of caries-affected dentin ${ }^{1,11}$. Moreover, there are no studies that demonstrate the influence of storage time and the type of substrate on GICs with incorporation of the $\mathrm{CHX}$ concentrations that were used in this study.

Thus, the purpose of this study was to evaluate the bond strength of a GIC associated with $\mathrm{CHX}$ to sound and caries-affected dentin, immediately and after six months of storage. The null hypotheses were that the bond strength of the GIC to dentin would not be altered by the condition of the substrate, concentration of $\mathrm{CHX}$ associated with $\mathrm{GIC}$, or storage time of the specimens.

\section{Material and Methods}

The experimental procedure flowchart is shown in Figure 1. Sample size was estimated using the PSPower and Sample Size Program software, version 3.0.43. The microshear bond strength values obtained in a pilot study were normally distributed with standard deviation 1.4 and the true difference between the experimental and control group mean values was 2.1. To be able to reject the null hypothesis with a power of $80 \%$ and a type I error probability of 0.05 it was necessary to have at least 8 specimens in each group. Considering possible losses during the experiment, 10 specimens were used in each group. 


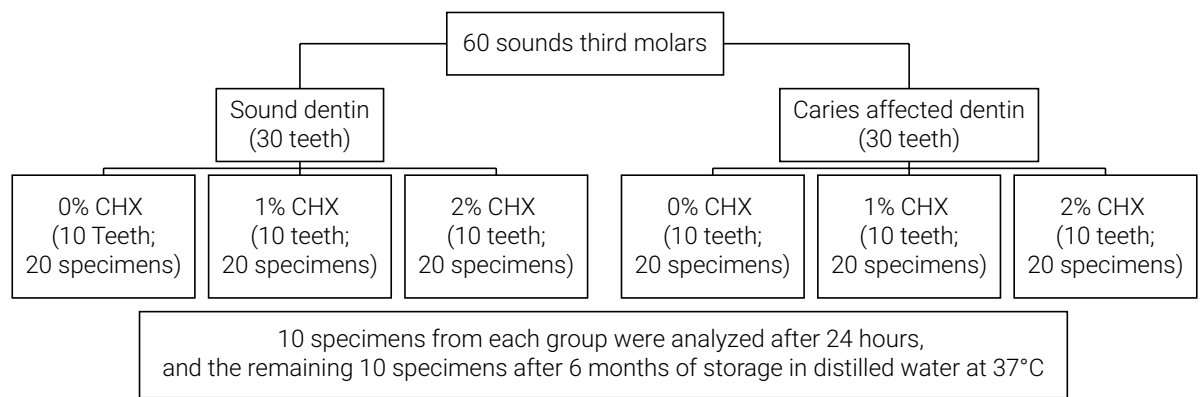

Figure. Flowchart of the distribution of groups

After approval by the Research Ethics Committee (Protocol 68.388), sixty sound extracted human third molars were obtained from the Tooth Bank of the Araraquara School of Dentistry - UNESP. Teeth without anatomical and structural defects were selected and a flat surface was produced in dentin by sectioning the teeth at the level of the occlusal third of the crown; by means of a diamond disc (No.11-4254, Buehler LTD., Lake Bluff, IL, USA) mounted in a metallographic cutter (ISOMET 1000, Buehler Ltd., Lake Bluff, IL, USA).

The teeth were divided into two groups of 30 teeth using simple random probabilistic sampling by chance. In one group they were submitted to artificial caries induction, and in the other dentin was maintained sound. The teeth allocated to the group with caries-affected dentin were sealed with two layers of acid resistant enamel, leaving only the dentin surface exposed. The specimens were sterilized with ethylene oxide, and then suspended in a cariogenic solution (BHI broth supplemented with $2 \%$ sucrose, $1 \%$ glucose and $0.5 \%$ yeast extract; $25 \mathrm{~mL} /$ tooth) inoculated with $10^{5} \mathrm{CFU} / \mathrm{mL}$ of Streptoccocus mutans ATCC 25175 (Tropical Culture Collection - André Toselo Foundation) (Figure 2A). The set was incubated under microaerophilic conditions at $37^{\circ} \mathrm{C}$ for 14 days. The cariogenic solution was changed every 48 hours, without inoculating new microorganisms. After this, the biofilm formed (Figure 2B) was removed, teeth were washed in deionized water. The resulting dentin surface was found to be darkened and softened when touched with a sharp explorer without pressure. Subsequently, the softened carious dentin (infected dentin) was manually removed using 320-grit silicon carbide abrasive paper under running water, until a touch resistant dentin (caries-affected dentin) was obtained ${ }^{15}$ (Figure 2C). The teeth with dentin surfaces that were maintained sound were also worn with the same type of abrasive paper, in an attempt to obtain a dentin depth similar to that of the caries-affected teeth.

The teeth were cleaned in an ultrasonic bath. Afterwards, they were embedded in self-polymerizing acrylic resin, by using a cylindrical PVC tube $(20 \mathrm{~mm} \times 18 \mathrm{~mm})$ as matrix, so that the dentin surface would be centralized and parallel to the base of the tube (Figure 2D).

Finally, the teeth of each group (sound dentin and caries-affected dentin) were reassigned, using simple random probabilistic sampling by chance, to three subgroups $(n=10)$ according to the chlorhexidine diacetate (Sigma Aldrish, Steinheim, Germany) concentration ( $0 \%, 1 \%$ and $2 \%$ by weight) added to the glass ionomer 
cement (Ketac Molar Easymix, 3M-ESPE Dental Products, St. Paul, MN, USA). Two specimens ( $1 \mathrm{~mm}$ diameter $\times 1 \mathrm{~mm}$ high) of the same material were prepared on the dentin surface of each tooth. Briefly, the bond area was delimited with double-faced acid resistant adhesive tape (3M Brazil, Sumaré, SP, Brazil) with a perforation measuring $1.0 \mathrm{~mm}$ in diameter (Figure 2E). Ketac Molar Liquid (3M - ESPE Dental Products, St. Paul, MN, USA) was applied to dentin for 10 seconds (Figure 2F), washed with a jet of water-air for 10 seconds and the dentin surface was dried with cotton wool balls. A cylindrical silicone matrix (Embramed, São Paulo, SP, Brazil) with an orifice $1 \mathrm{~mm}$ in diameter and $1 \mathrm{~mm}$ high was placed so that its internal diameter would coincide with the delimited adhesive area (Figure 2G).

The amount of $\mathrm{CHX}$ diacetate required to obtain final concentrations of $1 \%$ and $2 \%$ by weight was determined based on the average weight of a spoonful of the GIC powder. $\mathrm{CHX}$ diacetate was incorporated into the GIC powder immediately before manipulating the cement. The powder-liquid weight ratio recommended by the glass ionomer cement manufacturer was maintained. The material was manipulated at a room temperature of $24 \pm 1^{\circ} \mathrm{C}$, in accordance with the Manufacturer's recommendations (3M - ESPE Dental Products, St. Paul, MN, USA), and then inserted into the matrix with the aid of a Centrix syringe (DFL, Indústria e Comércio S.A, Jacarepaguá, RJ, Brazil) (Figure 2H). The tops of the test specimens were protected with Vaseline, and they were stored at $37^{\circ} \mathrm{C}$ in $100 \%$ humidity for 24 hours, avoiding their direct contact with the humidity. Subsequently, the matrixes were removed and the specimens were observed under a stereomicroscope (Olympus SZX7, Olympus Corporation, Tokyo, Japan) at 40x magnification to certify the absence of defects at the bond interface.

All the procedures were carried out by a single experience and previously trained operator.

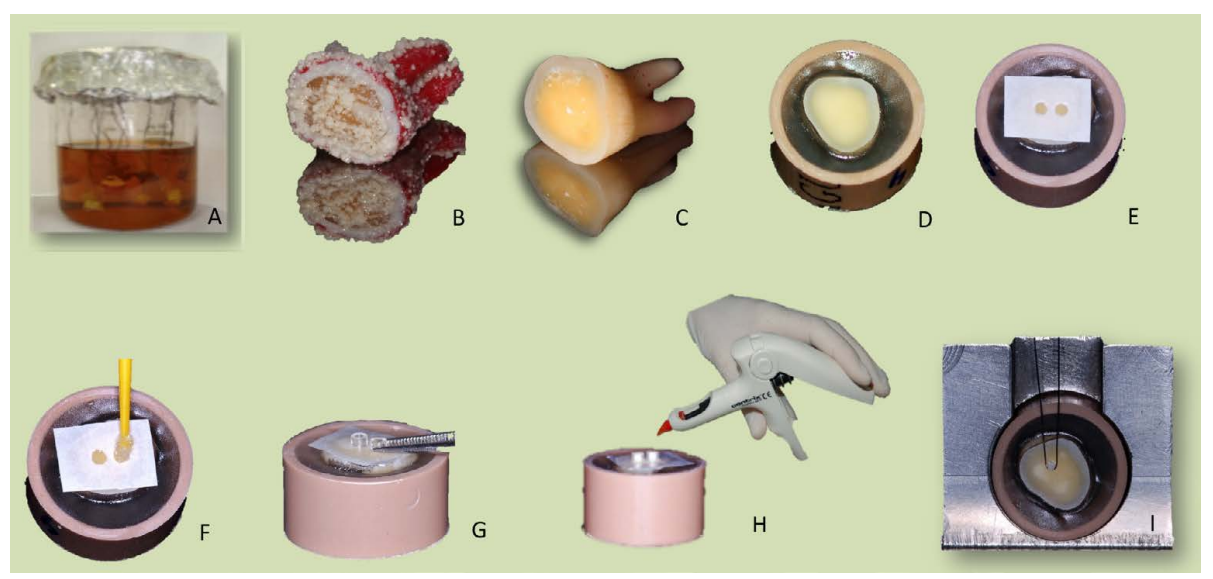

Figure 2. A: teeth immersed in cariogenic solution. B: tooth after 14 days of immersion in cariogenic solution. $\mathrm{C}$ : tooth after removal of infected dentin. D: tooth included in pvc tube with acrylic resin. E: delimitation of the adhesive area with double-sided tape. F: dentin conditioning with GIC liquid. G: positioning of the microtubes over the perforations in the adhesive tape. $\mathrm{H}$ : insertion of the materials into the microtubes. I: die positioned for the performance of the microshear test 


\section{Microshear bond strength test and failure mode analysis}

The microshear bond strength ( $\mu$ SBS) test was performed in one of the two specimens prepared on each tooth 24 hours after their fabrication, and the other specimen prepared on the same tooth was protected with Vaseline and remained stored in distilled water $(\mathrm{pH}=6.8-7.4)$ at $37^{\circ} \mathrm{C}$ for six months to evaluate the bond strength. During this period, distilled water $\mathrm{pH}$ was monitored once a week with the aid of a $\mathrm{pH}$ meter (Model Q400AS, Quimis Aparelhos Científicos, Diadema, SP, Brazil). In case of change in $\mathrm{pH}$, the distilled water was replaced with a fresh amount.

A mechanical testing machine (DL-Digital Line, EMIC, São José dos Pinhais, PR, Brazil), adjusted for tensile forces was used for the tests. A wire $0.2 \mathrm{~mm}$ in diameter was looped around the specimen as closely as possible to the interface (Figure 2I). Force was applied with a load cell of $100 \mathrm{~N}$ at a crosshead speed of $0.5 \mathrm{~mm} / \mathrm{min}$ until failure occurred, and the maximum stress values in MegaPascal (MPa) withstood by the dentin/material bond were recorded. Fractured surfaces were observed under a stereomicroscope (Olympus SZX7, Olympus Corporation, Tokyo, Japan) at 40x magnification. Failures were classified as adhesive (at substrate/restorative material interface), cohesive in dentin or in the material (fracture within the dentin or material, respectively), and mixed (partially adhesive and partially cohesive failures). Pre-testing failures were recorded, but were not included in the statistical analysis.

A single trained examiner, who did not know to which group each test specimen belonged, performed the $\mu \mathrm{SBS}$ test and the failure mode assessments.

\section{Statistical analysis}

The bond strength data (in MPa) passed the tests of normality (Shapiro Wilk; $p \geq 0.056$ ), but the assumption of homoscedasticity was not obeyed (Levene, $p=0.009$ ). Thus, three-way ANOVA was used and multiple comparisons, when necessary, were made by the Games-Howell post-test for heteroscedastic data. The Chi-square test was used to compare failure modes between conditions of substrate, storage periods and materials. All statistical tests were performed by the PASW Statistics software (v.22, SPSS Inc, Chicago, IL) and the level of significance adopted for decision-making was $5 \%$.

\section{Results}

The mean (SD) microshear bond strength ( $\mu$ SBS) values are shown in Table 1.

According to three-way ANOVA, the $\mu S B S$ values were significantly higher in sound dentin $(3.62 \pm 1.69 \mathrm{MPa})$ than in caries-affected dentin $(2.52 \pm 1.14 \mathrm{MPa})(p \leq 0.001$. Storage periods and different concentrations of $\mathrm{CHX}$ diacetate added to the $\mathrm{GIC}$, also had a significant influence on the $\mu$ SBS values ( $p=0.002$ and $p=0.001$, respectively), and there was a significant interaction between these two factors $(p \leq 0.036)$. Thus, the multiple comparisons showed that in sound dentin, $\mu \mathrm{SBS}$ of group $\mathrm{GIC}+\mathrm{CHX} 2 \%$ was statistically lower than the values found for Groups GIC and GIC + CHX $1 \%$ at 24 hours ( $p=0.005$ and $p=0.032$ respectively). In caries-affected dentin, $\mu \mathrm{SBS}$ in Group GIC $+1 \% \mathrm{CHX}$ at 24 hours was statistically higher than that observed for Groups $\mathrm{GIC}+2 \% \mathrm{CHX}$ at 24 hours ( $\mathrm{p}=0.001$ ) and $\mathrm{GIC}+1 \%$ $\mathrm{CHX}$ at 6 months $(\mathrm{p}=0.024)$. Irrespective of the substrate condition, comparisons showed no statistically significant differences between the other groups ( $p \geq 0.053)$. 
Table 1. Microshear bond strength ( $\mu$ SBS) of GIC to dentin, depending on the chlorhexidine diacetate concentration added and storage period

\begin{tabular}{|c|c|c|c|c|c|c|c|}
\hline \multirow{3}{*}{\multicolumn{2}{|c|}{ Substrate }} & \multirow{3}{*}{\multicolumn{2}{|c|}{ Material }} & \multicolumn{4}{|c|}{$\mu \mathrm{SBS}(\mathrm{MPa})^{\dagger}$} \\
\hline & & & & \multicolumn{4}{|c|}{ Storage period } \\
\hline & & & & \multicolumn{2}{|c|}{24 hours } & \multicolumn{2}{|c|}{6 months } \\
\hline \multirow{3}{*}{\multicolumn{2}{|c|}{ Sound Dentin }} & \multicolumn{2}{|c|}{ GIC } & \multicolumn{2}{|c|}{$4.5 \pm 1.4^{\mathrm{A}}$} & \multicolumn{2}{|c|}{$3.7 \pm 1.7^{\mathrm{AB}}$} \\
\hline & & \multicolumn{2}{|c|}{$\mathrm{GIC}+\mathrm{CHX} 1 \%$} & \multicolumn{2}{|c|}{$4.7 \pm 1.8^{\mathrm{A}}$} & \multicolumn{2}{|c|}{$3.4 \pm 1.7^{\mathrm{AB}}$} \\
\hline & & \multicolumn{2}{|c|}{$\mathrm{GIC}+\mathrm{CHX} 2 \%$} & \multicolumn{2}{|c|}{$2.4 \pm 0.9^{\mathrm{B}}$} & \multicolumn{2}{|c|}{$2.5 \pm 1.4^{\mathrm{AB}}$} \\
\hline \multirow{3}{*}{\multicolumn{2}{|c|}{ Caries-Affected Dentin }} & \multicolumn{2}{|c|}{ GIC } & \multicolumn{2}{|c|}{$3.3 \pm 1.2^{\mathrm{ab}}$} & \multicolumn{2}{|c|}{$2.1 \pm 1.0^{\mathrm{ab}}$} \\
\hline & & \multicolumn{2}{|c|}{$\mathrm{GIC}+\mathrm{CHX} 1 \%$} & \multicolumn{2}{|c|}{$3.6 \pm 0.7^{a}$} & \multicolumn{2}{|c|}{$1.9 \pm 1.1^{\mathrm{b}}$} \\
\hline & & \multicolumn{2}{|c|}{$\mathrm{GIC}+\mathrm{CH} \times 2 \%$} & \multicolumn{2}{|c|}{$2.0 \pm 0.6^{b}$} & \multicolumn{2}{|c|}{$2.1 \pm 1.2^{\mathrm{ab}}$} \\
\hline \multicolumn{8}{|c|}{$\begin{array}{l}\text { f- values correspond to mean and standard deviation } \\
\text { - different superscript capital letters } s^{(A, B)} \text { denote statistically significant differences between groups for sound } \\
\text { dentin (Games Howell test; } p \leq 0.032 \text { ) and different superscript lowercase letters }(\mathrm{a}, \mathrm{b}) \text { denote statistically } \\
\text { significant differences between groups for caries-affected dentin (Games Howell test; } p \leq 0.024 \text { ). }\end{array}$} \\
\hline & & & \multicolumn{5}{|c|}{ Failure modes } \\
\hline & & & $\begin{array}{l}\text { Cohesive in } \\
\text { material }\end{array}$ & Adhesive & Mixed & Pre-testing & $p$ value* \\
\hline \multirow{2}{*}{ Substrate } & \multicolumn{2}{|c|}{ Sound dentin } & $17(28.33)$ & $17(28.33)$ & $26(43.33)$ & $0(0.00)$ & \multirow{2}{*}{0.028} \\
\hline & Caries-aff & ntin & $27(45.00)$ & $7(11.67)$ & 19(31.67) & $7(11.67)$ & \\
\hline \multirow{2}{*}{$\begin{array}{l}\text { Storage } \\
\text { period }\end{array}$} & 24 hours & & $16(26.67)$ & $15(25.00)$ & $29(48.33)$ & $0(0.00)$ & \multirow{2}{*}{0.017} \\
\hline & 6 months & & $28(46.67)$ & $9(15.00)$ & $16(26.67)$ & $7(11.67)$ & \\
\hline \multirow{3}{*}{ Material } & $\mathrm{GIC}$ & & $16(40.00)$ & $7(17.50)$ & $13(32.50)$ & $4(10.00)$ & \multirow{3}{*}{0.633} \\
\hline & $\mathrm{GIC}+\mathrm{CH}\rangle$ & & $16(40.00)$ & $7(17.50)$ & $16(40.00)$ & $1(2.50)$ & \\
\hline & $\mathrm{GIC}+\mathrm{CH}\rangle$ & & $10(25.00)$ & $9(25.71)$ & $16(40.00)$ & $5(12.50)$ & \\
\hline
\end{tabular}

* Chi-square test results of failure mode proportions (significant difference: $p<0.05$ ).

Table 2 shows the percentage of failure modes and pre-testing failures. Only condition of substrate and storage period had significant influence on the failure modes ( $p=0.028$ and $p=0.017$ respectively). Irrespective of substrate condition and storage period, different concentrations of $\mathrm{CHX}$ diacetate showed no statistically significant influence on failure modes $(p=0.633)$. The following percentage of failure modes were found: $\mathrm{GIC}$ and $\mathrm{GIC}+\mathrm{CHX} 1 \%$ (40.0\% cohesive in material, $17.5 \%$ adhesive and $32.5 \%$ mixed), and GIC + CHX 2\% (25.0\% cohesive in material, $22.5 \%$ adhesive and $40.0 \%$ mixed) No cohesive failures in dentin were observed. Pre-testing failures were more frequent in caries-affected dentin and in the storage period of 6 months, and represented $10 \%$ in Group GIC, 2.5\% in Group GIC + CHX 1\% and 12.5\% in Group GIC + CHX 1\%.

\section{Discussion}

The findings of this study showed that the bond strength of the GIC to dentin was influenced by the condition of the substrate; concentration of $\mathrm{CHX}$ associated with $\mathrm{GIC}$, and storage time of the specimens, so the null hypotheses were rejected. Bonding to tooth structure is one of the most important mechanical properties of GICs ${ }^{9}$. Several factors may influence bond strength tests: test device; biological substrate; 
position of the specimens on the tooth, and storage time. In this study, the microshear bond strength test was performed, in which the area of the specimens is reduced and there is no great need to manipulate them during their preparation as there is in the microtensile bond strength test ${ }^{16}$.

The bond of GICs to caries-affected dentin is more complicated than to sound dentin because of its porosity and the presence of lactic acid involved in the caries lesion ${ }^{17,18}$ . Several studies have supported the use of artificially induced carious dentin to test new materials and techniques ${ }^{14,15,19}$, because it is difficult to standardize natural dentinal caries, since there are structural differences within the different carious zones. The size and shape of the naturally formed carious lesion are also characteristics that make it difficult to form a standardized flat substrate surface for bond strength tests. In addition, criteria commonly used to guide excavation of the lesion, such as color and tactile hardness, are subjective ${ }^{19}$.

In this study, the microbiological method of caries induction was applied to produce caries-affected dentin because lesions in dentin seem to have a molecular and structural arrangement that is more similar to that of natural lesions ${ }^{21}$.

Regarding storage time, in the present study only the group containing $\mathrm{CHX} 1 \%$ in period of 24 hours had $\mu$ SBS values that were statistically significant and higher than those of Group GIC + CHX 1\% after the 6-month period when the bond was produced in caries affected dentin. Colluci et al. ${ }^{13}$ (2014) analyzing the long-term water storage of GIC specimens observed a reduction in shear bond strength only when dentin surfaces were prepared with rotatory instruments. On the other hand, Azevedo et al. ${ }^{21}$ (2011) observed that after specimens were stored in water for 90 days, the bond strength improved, which was attributed to the acid-base reaction that occurs in a slow and continuous manner.

As observed in the present study, Group GIC $+2 \% \mathrm{CHX}$, showed a decrease in the bonding capacity to sound dentin in the 24-hour period compared with the Control Group and GIC $+1 \% \mathrm{CHX}$. Takahashi et al. ${ }^{22}$ (2006) also observed that in sound dentin the addition of $\mathrm{CHX}$ in concentration of $2 \%$ or higher caused a significant reduction in dentin bond strength after 24 hours of storage. Other antibacterial agents such as cetrimide, ciprofloxacin, metronidazole and minocycline associated with GICs at high concentrations have also shown decrease in bonding capacity of these materials $^{23,24}$. These results can be explained by the fact that a higher concentration of the antibacterial agent may interfere with the reaction between glass particles and liquid of the cement, thereby increasing the number of unreacted particles in the structure and reducing the mechanical properties ${ }^{24}$. For bond strength tests, it has also been hypothesized that antibacterial agents may affect the polar and ionic attraction force between carboxyl groups and inorganic ions in the dentin.

In a previous study Becci et al. ${ }^{25}$ (2014) compared the bond strength of a GIC with different concentrations of $\mathrm{CHX}$ to sound and caries-affected dentin only in the period of 24 hours. Although in both the cited study and the present study, the infected dentin was manually removed using 320-grit silicon carbide abrasive, the authors showed that the condition of the substrate had no influence on the immediate bond strength values. This was attributed to the excessive removal of carious dentin resulting in a 
caries-affected dentin with characteristics very close to those of sound dentin, and the loss of calcium ions was insufficient to determine bonding differences in the cited study. In the present study, the carious dentin was removed more carefully, taking care not to wear too much caries-affected dentin. The results showed that the bond strength values of the GIC to this dentine were statistically lower than those to sound dentin. No other studies using caries-affected dentin and antibacterial agents associated with GICs were found so that no comparison with this study could be made.

The literature has described that specimen storage in water at $37^{\circ} \mathrm{C}$ decreased the bond strength because water degrades the restorative material/dentin interface. An interesting finding of the present study was that despite the remarkably low bond strength values found for Group GIC $+2 \% \mathrm{CHX}$, the bond strength to caries-affected dentin did not decrease after 6 months, as it did for Groups GIC and $\mathrm{GIC}+1 \% \mathrm{CHX}$. This is an important result, since 6 months is a considerable period of the GIC restoration permanence on caries-affected dentin, depending on the clinical conditions found in the mouth. The addition of chlorhexidine to the material is particularly important when performing bonding to caries-affected dentin since chlorhexidine is an inhibitor of metalloproteinases and cysteine cathepsins ${ }^{26}$. Thus, addition of $2 \% \mathrm{CHX}$ could be considered a promising way to preserve the bond interface and increase the bond durability in clinical practice, as previously suggested ${ }^{14,27}$.

With respect to the failure modes, cohesive in material and mixed failures were predominant for all groups. The high frequency of cohesive failures within material were related to the low resistance of the tested material itself rather than its true bond strength to dentin ${ }^{16,29}$, and in the majority of instances this did not represent the real bond strength of the material to dentin ${ }^{29}$. A higher tendency towards cohesive fractures may also occur because of numerous porosities in the structure of the material, which may act as stress points ${ }^{30}$. In the present study, cohesive defects in material were observed in $25 \%$ to $40 \%$ of the specimens, and mixed fractures were present in $32.5 \%$ to $40 \%$ of the specimens for all materials irrespective of the substrate and storage time.

In summary, the current study demonstrated that time and substrate condition were important factors that should be considered when using GIC associated with antibacterial agents. Furthermore, although the addition of $2 \% \mathrm{CHX}$ affected the early bond strength, it seems to have protected the caries-affected dentin/ GIC bond during the storage period in water for 6 months.

Although the association of $\mathrm{CHX}$ with $\mathrm{GICs}$ seemed to be a promising alternative to increase their anticariogenic properties, further studies evaluating the physicochemical and antibacterial properties should be conducted with the purpose of determining the optimal concentration that would provide better antibacterial power and less degradation of the material over time.

\section{Acknowledgements}

The authors acknowledge the financial support provided by the Brazilian Federal Agency for the Support and Evaluation of Graduate Education 


\section{References}

1. Alves FB, Hesse D, Lenzi TL, Guglielmi Cde A, Reis A, Loguercio AD, et al. The bonding of glass ionomer cements to caries-affected primary tooth dentin. Pediatr Dent. 2013 Jul-Aug;35(4):320- 4.

2. Banerjee A, Kellow S, Mannocci F, Cook RJ, Watson TF. An in vitro evaluation of microtensile bond strengths of two adhesive bonding agents to residual dentine after caries removal using three excavation techniques. J Dent. 2010 Jun;38(6):480-9.

3. Almeida Neves A, Coutinho E, Cardoso MV, Lambrechts P, Van Meerbeek B. Current concepts and techniques for caries excavation and adhesion to residual dentin. J Adhes Dent. 2011 Feb;13(1):7-22.

4. Costa AR, Garcia-Godoy F, Correr-Sobrinho L, Naves LZ, Raposo LH, Carvalho FG, et al. Influence of Different Dentin Substrate (Caries-Affected, Caries-Infected, Sound) on Long-Term $\mu$ TBS. Braz Dent J. 2017 Jan-Feb;28(1):16-23.

5. Ngo H. Glass-ionomer cements as restorative and preventive materials. Dent Clin North Am. 2010 Jul;54(3):551-63.

6. Türkün LS, Türkün M, Ertuğrul F, Ateş M, Brugger S. Long-term antibacterial effects and physical properties of a chlorhexidine-containing glass ionomer cement. J Esthet Restor Dent. 2008;20(1):29-44

7. Tüzüner T, Kuşgöz A, Er K, Taşdemir T, Buruk K, Kemer B. Antibacterial activity and physical properties of conventional glass-ionomer cements containing chlorhexidine diacetate/cetrimide mixtures. $J$ Esthet Restor Dent. 2011 Feb;23(1):46-55.

8. Hoszek A, Ericson D. In vitro fluoride release and the antibacterial effect of glass ionomers containing chlorhexidine gluconate. Oper Dent. 2008 Nov-Dec;33(6):696-701.

9. Tyas MJ, Burrow MF. Adhesive restorative materials: a review. Aust Dent J. 2004 Sep;49(3):112-21.

10. Deepalakshmi M, Poorni S, Miglani R, Rajamani I, Ramachandran S. Indian. Evaluation of the antibacterial and physical properties of glass ionomer cements containing chlorhexidine and cetrimide: an in-vitro study. J Dent Res. 2010 Oct-Dec;21(4):552-6

11. Tenuta LM, Ribeiro CC, Goncalves NC, Del Bel Cury AA, Aires CP, Tengan C, et al. The shortterm in situ model to evaluate the anticariogenic potential of ionomeric materials. J Dent. 2005 Jul;33(6):491-7

12. Reis KR, Spyrides GM, Oliveira JA, Jnoub AA, Dias KR, Bonfantes G. Effect of cement type and water storage time on the push-out bond strength of a glass fiber post. Braz Dent J. 2011;22(5):359-64

13. Colucci V, de Araújo Loiola AB, da Motta DS, do Amaral FL, Pécora JD, Corona SA. Influence of long term water storage and thermocycling on shear bond strength of glass-ionomer cement to Er:YAG laser-prepared dentin. J Adhes Dent. 2014 Feb;16(1):35-9.

14. Sanabe ME, Costa CA, Hebling J. Exposed collagen in aged resin-dentin bonds produced on sound and caries-affected dentin in the presence of chlorhexidine. J Adhes Dent. 2011 Apr;13(2):117-24

15. Ricci HA, Scheffel DL, de Souza Costa CA, dos Santos FJ, Jafelicci M Jr, Hebling J. Wettability of chlorhexidine treated non-carious and caries-affected dentin. Aust Dent J. 2014 Mar;59(1):37-42.

16. Bonifácio CC, Shimaoka AM, de Andrade AP, Raggio DP, van Amerongen WE, de Carvalho RC. Micromechanical bond strength tests for the assessment of the adhesion of GIC to dentine. Acta Odontol Scand. 2012 Dec;70(6):555-63.

17. Czarnecka B, Deregowska-Nosowicz P, Limanowska-Shaw H, Nicholson JW. Shear bond strengths of glass-ionomer cements to sound and to prepared carious dentine. J Mater Sci: Mater Med. 2007 May; 18(5):845-9.

18. Nicholson JW, Aggarwal A, Czarnecka B, Limanowska-Shaw H. The rate of change of pH of lactic acid exposed to glass-ionomer dental cements. Biomaterials. 2000 Oct;21(19):1989-93. 
19. Lenzi TL, Tedesco TK, Calvo AF, Ricci HA, Hebling J, Raggio DP. Does the method of caries induction influence the bond strength to dentin of primary teeth? J Adhes Dent. 2014 Aug;16(4):333-8.

20. Pacheco LF, Banzi EV, Rodrigues E, Soares LE, Pascon FM, Correr-Sobrinho L, et al. Molecular and structural evaluation of dentin caries-like lesions produced by different artificial models. Braz Dent J. 2013 Nov-Dec;24(6):610-8.

21. Azevedo ER, Coldebella CR, Zuanon AC. Effect of ultrasonic excitation on the microtensile bond strength of glass ionomer cements to dentin after different water storage times. Ultrasound Med Biol. 2011 Dec;37(12):2133-8.

22. Takahashi Y, Imazato S, Kaneshiro AV, Ebisu S, Frencken JE, Tay FR. Antibacterial effects and physical properties of glass-ionomer cements containing chlorhexidine for the ART approach. Dent Mat. 2006 Jul;22(7):647-52.

23. Prabhakar AR, Prahlad D, Kumar SR. Antibacterial activity, fluoride release, and physical properties of an antibiotic-modified glass ionomer cement. Pediatr Dent. 2013 Sep-Oct;35(5):411-5.

24. Yesilyurt C, Er K, Tasdemir T, Buruk K, Celik D. Antibacterial activity and physical properties of glassionomer cements containing antibiotics. Oper Dent. 2009 Jan- Feb;34(1):18-23.

25. Becci ACO, Marti LMM, Zuanon ACC, Brighenti FL, Giro EMA. Influence of the addition of chlorhexidine diacetate on bond strength of a high-viscosity glass ionomer cement to sound and artificial caries-affected dentin. Rev Odontol UNESP. 2014;43(1):1-7.

26. Hebling J, Pashley DH, Tjäderhane L, Tay FR. Chlorhexidine Arrests Subclinical Degradation of Dentin Hybrid Layers in vivo. J Dent Res. 2005 Aug;84(8):741-6.

27. Tjäderhane L, Nascimento FD, Breschi L, Mazzoni A, Tersariol IL, Geraldeli S, et al. Strategies to prevent hydrolytic degradation of the hybrid layer-A review. Dent Mater. 2013;29(10):999-1011.

28. Peez R, Frank S. The physical-mechanical performance of the new Ketac Molar Easymix compared to commercially available glass ionomer restoratives. J Dent. 2006 Sep;34(8):582-7.

29. Choi K, Oshida Y, Platt JA, Cochran MA, Matis BA, Yi K. Microtensile bond strength of glass ionomer cements to artificially created carious dentin. Dent. 2006 Sep-Oct;31(5):590-7.

30. Hoshika S, De Munck J, Sano H, Sidhu SK, Van Meerbeek B. Effect of conditioning and aging on the bond strength and interfacial morphology of glass-ionomer cement bonded to dentin. J Adhes Dent. 2015;17(2):141-6 